\title{
Attempts to demonstrate magnetic discrimination by homing pigeons in flight
}

\author{
GEORGE J. CARMAN, MICHAEL M. WALKER, and ANDY K. LEE \\ California Institute of Technology, Pasadena, California
}

\begin{abstract}
Eight homing pigeons, trained to fly between two elevated feeders within a flight tunnel, were tested for their ability to discriminate between two magnetic field stimuli and two acoustic stimuli, using a unitary discrete-trials procedure with successive presentation of stimuli. Magnetic stimuli consisted of the ambient magnetic field and a reduced magnetic field in which the vertical component of the field was reduced to $50 \%$ of its ambient value. Acoustic stimuli consisted of an ambient white noise and the white noise plus a tone. Stimuli were paired with food reward and either a time penalty (Experiment 1) or electric shock (Experiment 2). Although subjects could discriminate sounds with our procedures, none of the subjects demonstrated discrimination of magnetic fields. The failure of pigeons to discriminate magnetic stimuli is discussed as a consequence of either the failure to provide conditions sufficient for such discrimination or the absence of a magnetic sense in these animals.
\end{abstract}

Although behavioral experiments in the field have suggested the use of a magnetic sense in the navigation of homing pigeons (Frei \& Wagner, 1976; Keeton, 1971; Walcott, 1978; Walcott \& Green, 1974), various attempts to demonstrate sensitivity to magnetic stimuli in the laboratory have failed (Alsop, 1987; Beaugrand, 1976; Delius \& Emmerton, 1978; Griffin, 1982; Kreithen \& Keeton, 1974; McIsaac \& Kreithen, 1987; Meyer \& Lambe, 1966; Moore, Stanhope, \& Wilcox, 1987; Orgel \& Smith, 1954). If a magnetic sense exists in these animals, such failures to demonstrate magnetic discrimination in the laboratory could be due to the failure to satisfy any one of several prerequisites for such a demonstration. The suggestion that flight may be necessary for discrimination of magnetic stimuli by pigeons (Kreithen \& Keeton, 1974) might explain the lack of discrimination of magnetic stimuli in conventional keypecking or cardiac conditioning paradigms. Such an explanation was supported by the experiments of Bookman (1977), who reported that mated pairs of homing pigeons discriminated magnetic stimuli only when they "fluttered" (performed sustained hovering, jumping, rapid turning, or short flights) before entering one or the other of two goalboxes located at one end of a flight tunnel.

We began our investigations with an attempt to replicate the experiments of Bookman (1977), duplicating his apparatus and using similar magnetic stimuli and proce-

\footnotetext{
We thank J. Kirschvink and M. Konishi of the California Institute of Technology for providing laboratory space and partial support for these experiments, and M. E. Bitterman of the University of Hawaii for valuable advice and criticism. We also thank Michelle Mahowald for assisting in the construction of the apparatus and in the replication of Bookman's experiments, and Senior Instrument Specialist Herb Adams of Caltech's Central Engineering Services for the design and construction of the feeder platforms. This research was supported in part by NIH BRSG Grant RR07003 and by an NSF Graduate Fellowship to G.J.C. The authors' mailing address is: Division of Biology 216-76, California Institute of Technology, Pasadena, CA 91125.
}

dures (M. A. Bookman, personal communication, 1983). Two mated pairs of homing pigeons were first pretrained to allow for adaptation to the flight tunnel and shaping of their responses. During the subsequent magnetic discrimination training, we observed only random performance over a course of 307 trials for one pair and 149 trials for the other pair (Carman \& Mahowald, 1984). Random performance was observed regardless of whether we examined all responses made by each pair or only the responses made during trials accompanied by the "fluttering' 'behavior that Bookman (1977) had claimed to be correlated with discrimination of magnetic stimuli.

Next we attempted control experiments using a variety of light and sound stimuli to determine whether the twochoice paradigm used by Bookman was capable of demonstrating discrimination via known sensory modalities. To facilitate data collection, we automated the experiments, thereby eliminating the handling of birds by experimenters and the associated possibility of inadvertent cues. In addition, we used individual homing pigeons, rather than mated pairs of birds, the latter having often been observed to engage in a variety of behaviors other than the desired goal-directed response. Although we observed discrimination of discrete light stimuli contiguous with the goalboxes and discrimination of diffuse light stimuli presented between the goalboxes, we did not observe discrimination of either discrete, contiguous sound stimuli or diffuse sound stimuli in any of 4 birds tested (Carman \& Mahowald, 1984). The failure of this paradigm to demonstrate discrimination of such known sensory stimuli raised serious doubts about its suitability for demonstrating the existence of a hypothetical magnetic sense.

Such discriminative choice experiments, in which stimuli are presented successively, with only one stimulus present during each trial, are difficult discrimination tasks and frequently fail with well-understood, salient stimuli (Bitterman, 1979; Mackintosh, 1974). The failure 
of the above experiments to produce discrimination not only of magnetic stimuli but also of acoustic stimuli led us to employ a unitary discrimination training procedure developed by Woodard and Bitterman (1974) in our subsequent experiments. Such unitary procedures, in which the rate of a single response to each of two successively presented stimuli is measured, yield discrimination more readily than choice procedures (Mackintosh, 1974), while requiring multiple responses during trials improves discrimination (Bitterman, 1979). In our experiments, individually trained birds were required to shuttle repeatedly between two cylindrical feeders positioned at opposite ends of a flight tunnel during each trial. Depending on the magnetic field stimulus present during a trial, this response was either rewarded with food or punished with a time penalty (Experiment 1) or electric shock (Experiment 2) at the end of the trial.

\section{MATERIALS AND METHODS}

\begin{abstract}
Apparatus
The present experiments were performed in an elevated flight tunnel (interior measurements: $1.07 \mathrm{~m}$ wide $\times 0.89 \mathrm{~m}$ high $\times$ $3.40 \mathrm{~m}$ long) constructed from wood and nonferrous hardware (Figure 1). Within the tunnel were located two cylindrical feeders (20.3 cm in diameter and $40.6 \mathrm{~cm}$ high), also constructed of nonferrous materials. Atop each feeder was a spring-supported platform coupled to three microswitches wired in series, which allowed for the automatic collection of data. Each feeder contained a pneumatic piston-driven timed-access feeder that delivered split peas through a central hole $(2.54 \mathrm{~cm}$ in diameter $)$ located at the center of each platform and equipped with an infrared photobeam to detect feeding. Atop each platform were a set of eight concentric aluminum rings connected to a remote high-voltage pulse generator that could deliver trains of constant power shocks to the feet of the pigeons while they stood atop the feeder. The interior of the tunnel
\end{abstract}

was illuminated during trials by two lamps powered by a remote $12-\mathrm{V} \mathrm{dc}$ supply, providing an average luminance of $1.6 \mathrm{fL}$. Access to the interior of the tunnel was provided through hinged side panels, three of which made up each side.

For magnetic discrimination training, the magnetic field within the tunnel was manipulated by three pairs of circular coils, each coil consisting of 150 turns of 22-ga wire wound on a 1.11-m-diam styrofoam core (Figure 1). The coils were powered by a customdesigned three-channel current source capable of producing steady state magnetic fields with less than \pm 11 gamma of noise (1 gamma $=10^{-5} \mathrm{G}$ ). Using this apparatus, we produced two magnetic field conditions for use as stimuli: (1) the ambient magnetic field (AMF) due to the local geomagnetic field, and (2) the reduced magnetic field (RMF), in which the field produced by the coils reduced the vertical component of the local geomagnetic field to approximately $50 \%$ of its normal value. In contrast, the horizontal component of the magnetic field for the two conditions differed on the average by less than $1 \%$ in magnitude and in direction (see Table 1). Measurements of the magnetic field were made with a Develco fluxgate magnetometer (Model 9200C) and were accurate to within $\pm 1 \%$.

During both magnetic and acoustic discrimination training, white noise was used to mask environmental sounds throughout the sessions. The white noise was delivered from a speaker centered beneath the flight tunnel at an intensity of $75 \mathrm{~dB}$ SPL measured in the flight tunnel. For acoustic discrimination training, we also used a tone with audible harmonic distortion. This tone was produced by driving a second speaker centered beneath the flight tunnel with a $440-\mathrm{Hz}$ square wave at an intensity of $95 \mathrm{~dB}$ SPL measured in the flight tunnel. Two acoustic conditions were used as stimuli: (1) the ambient sound consisting of the white noise (AS), and (2) the altered sound consisting of the white noise and the tone (RS).

A microcomputer located in a nearby room controlled all aspects of the experimental procedure, including the timing of trials and intertrial intervals, the sequence of magnetic or acoustic stimuli, the delivery of reward and punishment, and the automatic record ing of behavior. A closed-circuit television camera (Sony AVC3260 ) allowed us to monitor and record the activity of the pigeons within the flight tunnel on video cassettes to provide an auxiliary record of behavior.

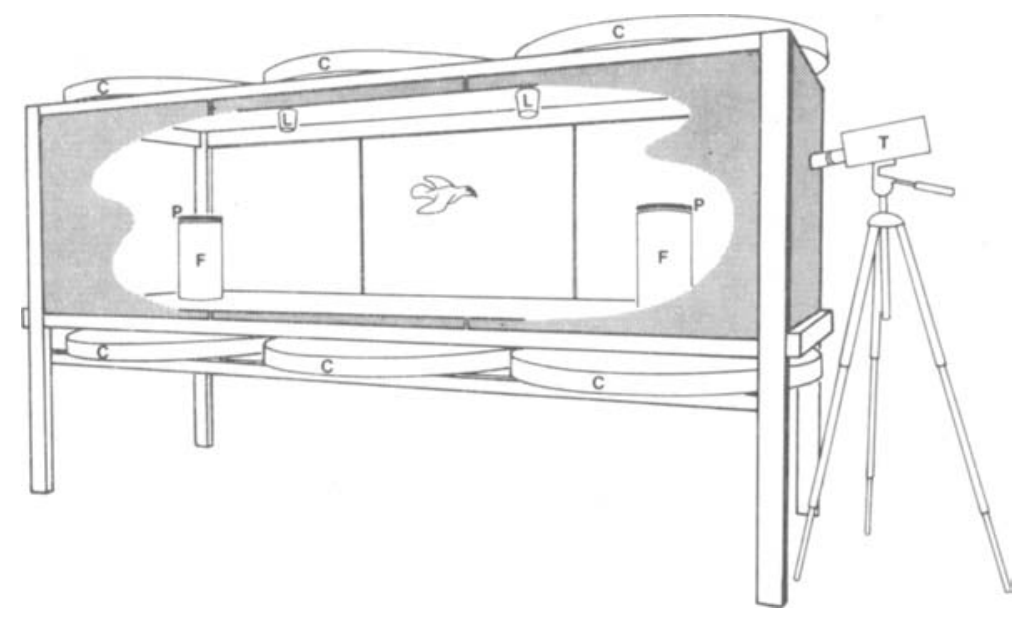

Figure 1. Perspective view of the flight tunnel used in the present experiments. Portions of the side panels have been cut away to reveal the interior. F, cylindrical feeders; $P$, platforms atop feeders with concentric aluminum rings (not shown) for the delivery of electric shock; $C$, coils for the control of magnetic stimuli; $L$, tunnel lights; and $T$, television camera. See text for details. 
Table 1

Mean and Standard Deviation of Magnetic Field Components as a Function of Magnetic Field Condition

\begin{tabular}{|c|c|c|c|c|c|c|}
\hline \multirow{3}{*}{$\begin{array}{l}\text { Magnetic } \\
\text { Field } \\
\text { Condition }\end{array}$} & \multicolumn{4}{|c|}{ Horizontal Component } & \multirow{2}{*}{\multicolumn{2}{|c|}{$\begin{array}{c}\text { Vertical Component } \\
\begin{array}{c}\text { Magnitude } \\
\text { (gamma) }\end{array}\end{array}$}} \\
\hline & \multicolumn{2}{|c|}{$\begin{array}{l}\text { Magnitude } \\
\text { (gamma) }\end{array}$} & \multicolumn{2}{|c|}{$\begin{array}{l}\text { Direction } \\
\text { (degrees) }\end{array}$} & & \\
\hline & Mean & $S D$ & Mean & $S D$ & Mean & $S D$ \\
\hline $\begin{array}{c}\text { AMF } \\
\text { RMF } \\
\text { [AMF-RMF] }\end{array}$ & $\begin{array}{r}20,010 \\
19,820 \\
192\end{array}$ & $\begin{array}{r} \pm 2,870 \\
\pm 2,470 \\
\pm 534\end{array}$ & $\begin{array}{c}69.2 \\
68.9 \\
0.35\end{array}$ & $\begin{array}{l} \pm 3.11 \\
\pm 3.51 \\
\pm 1.63\end{array}$ & $\begin{array}{l}-40,250 \\
-19,700 \\
-20,550\end{array}$ & $\begin{array}{l} \pm 1,580 \\
\pm 2,540 \\
\pm 1,450\end{array}$ \\
\hline
\end{tabular}

Note - Means and standard deviations were calculated for 10 measurements taken at equidistant locations along the flight path from one feeder to the other under each magnetic field condition (AMF and RMF), and for the 10 differences in each measurement between the two conditions ([AMF-RMF]). Note that 1 gamma $=10^{-5} \mathrm{G}$. The direction of the horizontal component is given relative to the long axis of the tunnel, as measured counterclockwise from the TV camera end when viewed from above.

\section{Subjects and Experimental Procedure}

The subjects were 8 experimentally naive homing pigeons of the gray check and wine check varieties, aged 6 to 24 months. All birds had been raised at the local Caltech loft and were given the opportunity for daily flight experience. All birds had demonstrated their homing ability in at least three homing trials over distances of at least $8 \mathrm{~km}$.

The subjects were isolated in individual cages, given free access to water and grit, and switched from their normal mixed-grain diet to limited amounts of split peas. Pretraining began when the birds were reduced to about $90 \%$ of their ad-lib weights. With the flight tunnel darkened, each bird was placed upon the feeders, which were initially located together at the midpoint of the flight tunnel. When the tunnel was illuminated, a few split peas placed near the central hole in the feeder platform attracted the attention of the birds to the hole. The feeder mechanisms then were operated alternately, leading the birds back and forth from one feeder to the other. As the distance between the feeders was progressively increased over a period of days, the birds rapidly learned first to step, then to hop, and finally to fly between the feeders. During this time, the birds also learned to mount the feeders from the floor of the tunnel.

Once each bird had learned to shuttle between the feeders, the trial procedure was incorporated into the pretraining. Each daily session consisted of 10 trials, each trial beginning with the illumination of the tunnel and the presentation of the magnetic field associated with reinforcement ( $\mathrm{S}+$ ). During each trial, each bird flew back and forth between the feeders, with each complete shuttle (i.e., leaving one feeder and arriving at the other) being counted as a response. At the first response after $90 \mathrm{sec}$, the bird was given $15 \mathrm{sec}$ access to food, after which the trial was terminated, the tunnel lights turned off, and the AMF continued or restored for the duration of the $210-\mathrm{sec}$ intertrial interval. This procedure continued until each bird had completed at least 3 days of pretraining with the feeders separated by $229 \mathrm{~cm}$, the distance used during discrimination testing. Body weight was maintained at a fixed percentage $( \pm 5 \mathrm{~g})$ between $70 \%$ and $80 \%$ of ad lib for each bird by supplemental feeding when necessary after each session.

Experiment 1. During discrimination training, each bird was given 20 trials in each daily session, 10 with the reinforced stimulus $(\mathrm{S}+)$ and 10 with the nonreinforced stimulus $(\mathrm{S}-)$. Stimuli were presented in a balanced, quasi-random order (Gellermann, 1933). Training was balanced for stimuli, with 2 birds receiving AMF as $\mathrm{S}+$ and $\mathrm{RMF}$ as $\mathrm{S}-$ and 2 birds receiving $\mathrm{RMF}$ as $\mathrm{S}+$ and $\mathrm{AMF}$ as $\mathrm{S}-$. On $\mathrm{S}+$ trials, at the first response after $90 \mathrm{sec}$, the bird was given $15 \mathrm{sec}$ access to food, after which the trial was terminated. For $\mathbf{S}-$ trials, the bird was given a time penalty whose duration was determined by a timer that was reset by subsequent responses either until the bird ceased responding for $15 \mathrm{sec}$, thus terminating the trial, or until a total of $180 \mathrm{sec}$ of penalty time had accrued, at which time the trial was terminated. During the intertrial interval, whose duration was varied pseudorandomly from 30 to $90 \mathrm{sec}$ with a mean of $60 \mathrm{sec}$, the tunnel lights were out and the AMF was present.

Experiment 2. Discrimination training of 4 additional birds proceeded as in Experiment 1 except for the substitution of electric shock for the time penalty during $S-$ trials. For these trials, responses after $90 \mathrm{sec}$ resulted in an electric shock to the bird for $15 \mathrm{sec}$ or for as long as it remained atop the conductive rings on either feeder platform, after which the trial was terminated. Alternatively, no shock was given if no responses occurred between 90 and $105 \mathrm{sec}$, at which time the trial was terminated.

At least 10 sessions (200 trials) of magnetic field discrimination training were followed by at least 10 sessions ( 200 trials) of acoustic discrimination training as a control for both Experiment 1 and Experiment 2. The procedures used for acoustic discrimination training were identical to those used for magnetic discrimination training, except that the magnetic stimuli were replaced by the acoustic stimuli (AS replaced AMF and RS replaced RMF). The AMF was present in the tunnel throughout these control experiments.

We chose this stimulus order (first magnetic, then acoustic) in order to avoid the possibility that experience with the ambient magnetic field during both $\mathrm{S}+$ and $\mathrm{S}$ - acoustic discrimination trials might interfere with subsequent magnetic discrimination training had the alternative stimulus order been chosen. Since the acoustic discrimination training was performed only as a test of the effectiveness of our procedures, we did not attempt to balance the order of the two stimulus types, but chose instead to optimize the conditions for magnetic discrimination. Although this resulted in a confounding of the stimulus type (magnetic or acoustic) with the stimulus order (first or second), we considered it extremely unlikely that discrimination would depend on the order of the stimuli rather than on the type of stimulus used.

\section{RESULTS}

\section{Experiment 1}

Data consisted of the number of responses within the first $90 \mathrm{sec}$ of each trial during the last 10 sessions of magnetic discrimination training and the last 10 sessions of acoustic discrimination training for each subject. Using these data, we calculated the mean and standard error for both the $\mathbf{S}+$ and the $S-$ trials of each session (Figure 2). These data were also used in an analysis of variance (BMDP8V ANOVA, equal cell size and mixed models, 


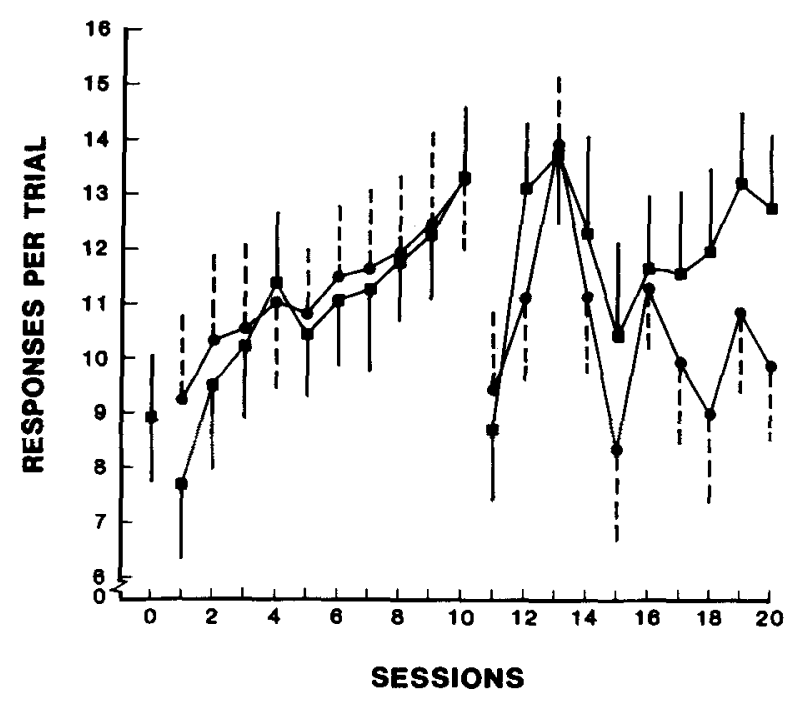

Figure 2. Mean and standard error of the number of responses for $\mathbf{S}+$ trials (squares and solid error bars) and $\mathbf{S}$ - trials (circles and dashed error bars) for the 4 subjects given a time penalty as punishment on S- (Experiment 1). Each point represents the mean of 10 trials from a single daily session for each of 4 subjects. Session 0: last day of pretraining (using $S+$ alone). Sessions 1 to 10 : magnetic diserimination training. Sessions 11 to 20: acoustic discrimination training.

BMDP Statistical Software, Inc., Los Angeles, CA 90025) in order to examine the main effects and interactions of various factors in our experimental design (Table 2).

No bird demonstrated discrimination of the magnetic stimuli (Figure 2, Sessions 1 to 10). This is confirmed in the analysis of variance by the absence of a significant main effect of reinforcement and the absence of a significant interaction of reinforcement with sessions (experience), which would be expected if subjects had discriminated the stimuli and learned their association with reinforcement or nonreinforcement (Table 2, sources $R$ and $\mathrm{R} \times \mathrm{Se}$ ). The only significant effect found for mag-

Table 2

Results of ANOVA for Experiment 1

\begin{tabular}{|c|c|c|c|c|c|}
\hline \multirow[b]{2}{*}{ Source } & \multirow[b]{2}{*}{$d f$} & \multicolumn{2}{|c|}{ Magnetic Stimuli } & \multicolumn{2}{|c|}{ Acoustic Stimuli } \\
\hline & & $F$ & $p$ & $F$ & $p$ \\
\hline $\mathbf{R}$ & 1 & 1.30 & 0.3718 & 9.30 & 0.0928 \\
\hline St & 1 & 0.01 & 0.9298 & 0.08 & 0.8010 \\
\hline $\mathrm{Se}$ & 9 & 2.58 & $0.0413^{*}$ & 1.06 & 0.4319 \\
\hline $\mathbf{R} \times \mathbf{S t}$ & 1 & 0.01 & 0.9452 & 0.08 & 0.8070 \\
\hline $\mathbf{R} \times \mathbf{S e}$ & 9 & 0.56 & 0.8117 & 3.37 & $0.0136^{*}$ \\
\hline St $\times$ Se & 9 & 1.73 & 0.1550 & 0.66 & 0.7351 \\
\hline $\mathrm{R} \times \mathrm{St} \times \mathrm{Se}$ & 9 & 0.39 & 0.9247 & 1.56 & 0.2008 \\
\hline
\end{tabular}

Note-Key to source: $\mathrm{R}=$ main effect of reinforcement (reward or punishment); $S t=$ main effect of stimuli (AMF or RMF; AS or RS); $\mathrm{Se}=$ main effect of sessions (experience) $\mathrm{R} \times \mathrm{St}=$ interaction of reinforcement with stimuli; $R \times S e=$ interaction of stimuli with sessions (learning); $\mathrm{St} \times \mathrm{Se}=$ interaction of stimuli with sessions; $\mathbf{R} \times \mathbf{S t} \times \mathbf{S e}=$ interaction of reinforcement with stimuli and sessions (interaction of stimulus conditions and learning). *Probabilities less than the .05 criterion value, indicating significant main effects or interactions. netic discrimination training was a steady increase in the rate of responses to both magnetic stimuli during the course of training (Table 2, source Se). Such an effect would be expected if the birds had not reached their maximum rates of response by the beginning of discrimination training.

In contrast, comparison of the number of responses for $S+$ and $S-$ trials over the course of acoustic discrimination training shows a progressive separation of the response rates to the two acoustic stimulus conditions (Figure 2, Sessions 11 to 20). Although the analysis of variance of these data revealed no significant main effect of reinforcement, there is a significant interaction of reinforcement with sessions (experience) (Table 2, sources $R$ and $R \times S e$ ). This result would be expected in the case of discrimination that is acquired partway through the course of training.

\section{Experiment 2}

As was observed in Experiment 1, no bird in Experiment 2 demonstrated discrimination of the magnetic field stimuli (Figure 3, Sessions 1 to 10). In contrast, however, we observed a decline in the rate of response to both magnetic stimulus conditions during training. We attributed this decline in response rate to an avoidance of the feeders due to the experience of shock. However, neither this decline in rate nor any other effect proved statistically significant in the analysis of variance (Table 3 ).

Separation of the response rates to the reinforced and punished acoustic stimulus conditions as well as a partial recovery of response rates were observed in the second half of Experiment 2 (Figure 3, Sessions 11 to 20). Two of the birds (those given AS as S+ and RS as S-) achieved and maintained discrimination of the acoustic stimuli to the end of training. The remaining 2 birds (those given RS as $\mathbf{S +}$ and AS as $\mathbf{S}-$ ) initially achieved discrimination, but failed to maintain this discrimination due to a feeder malfunction, during Session 17, which prevented the delivery of the reinforcement under $\mathbf{S}+$. The effect of this malfunction can be observed in the mean of the rates of response of all 4 birds (Figure 3, Session 17). Although this malfunction occurred for only one session, it resulted in a markedly reduced separation of the response rates under the two acoustic stimulus conditions for the remainder of the sessions for these 2 birds.

These results are reflected in the analysis of variance of the data, which showed neither a stimulus main effect nor a learning interaction (Table 3 , sources $R$ and $R \times$ Se). There was, however, a significant interaction of reinforcement, stimuli, and experience (Table 3 , source $R \times$ $\mathrm{St} \times \mathrm{Se})$. Although this interaction could be interpreted as an effect of the pairing of stimuli with reinforcement upon learning during training, it probably represents contamination of a learning effect by the decreased performance of the 2 birds that experienced the feeder malfunction, both of which were given the same pairing of stimuli and reinforcement. 


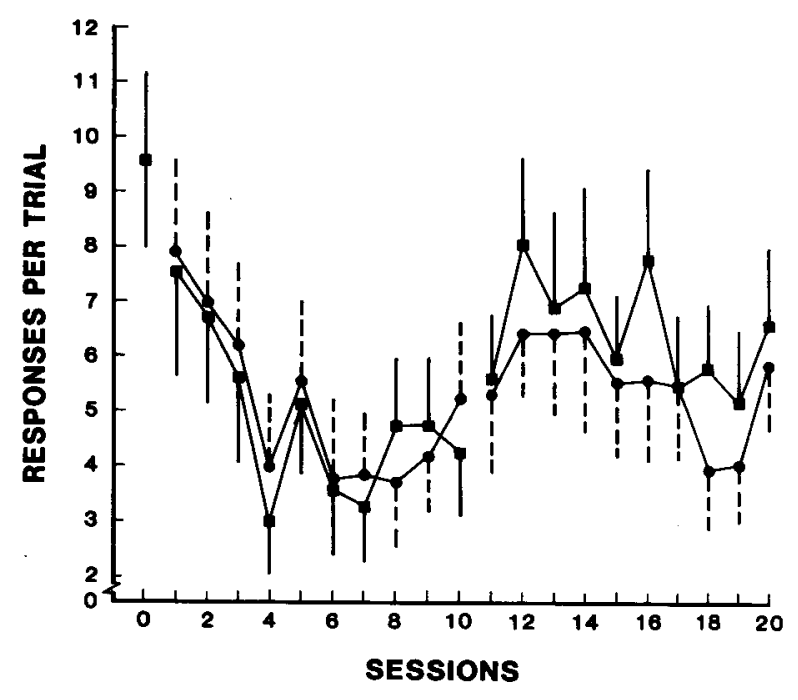

Figure 3. Mean and standard error of the number of responses for $\mathbf{S}+$ trials (squares and solid error bars) and $S-$ trials (circles and dashed error bars) for the 4 subjects given an electric shock as punishment on S- (Experiment 2). Each point represents the mean of 10 trials from a single daily session for each of $\mathbf{4}$ subjects. Session 0: last day of pretraining (using $S+$ alone). Sessions 1 to 10: magnetic discrimination training. Sessions 11 to 20: acoustic discrimination training.

\section{DISCUSSION}

A successful demonstration of sensory discrimination depends on the simultaneous satisfaction of a number of prerequisites, including (1) the use of a species suspected of possessing the sensory modality in question, (2) the selection of sensory stimuli thought to be discriminable to the animal, (3) the determination of a conditioned response that the animal is capable of associating with such stimuli, and (4) the design of experimental apparatus and procedures that allow both for the detection of stimuli and for their association to the conditioned behavioral response. The failure to demonstrate discrimination of magnetic field stimuli in the present experiments could be due to one or more of these prerequisites' not having been satisfied, a possibility for which there are precedents in other sensory modalities of the pigeon (Kreithen, 1978; Ossenkopp \& Barbeito, 1978). The results of our control

Table 3

Results of ANOVA for Experiment 2

\begin{tabular}{lcccccc}
\hline & & \multicolumn{2}{c}{ Magnetic Stimuli } & & \multicolumn{2}{c}{ Acoustic Stimuli } \\
\cline { 3 - 4 } \cline { 5 - 6 } Source & $d f$ & $F$ & $p$ & & $F$ & $p$ \\
\hline $\mathbf{R}$ & 1 & 1.02 & 0.4183 & & 6.67 & 0.1229 \\
$\mathrm{St}$ & 1 & 0.01 & 0.9217 & & 0.11 & 0.7749 \\
$\mathrm{Se}$ & 9 & 0.96 & 0.4995 & & 1.08 & 0.4201 \\
$\mathrm{R} \times \mathrm{St}$ & 1 & 0.13 & 0.7546 & & 0.18 & 0.7152 \\
$\mathrm{R} \times \mathrm{Se}$ & 9 & 0.93 & 0.5244 & & 1.50 & 0.2207 \\
$\mathrm{St} \times \mathrm{Se}$ & 9 & 0.45 & 0.8899 & & 1.13 & 0.3895 \\
$\mathrm{R} \times \mathrm{St} \times \mathrm{Se}$ & 9 & 1.09 & 0.4191 & & 3.05 & $0.0212^{*}$ \\
\hline
\end{tabular}

Note-See Table 2 for key to source. experiments, which used diffuse acoustic stimuli, suggest that our design of apparatus and our procedures were adequate for demonstrating discrimination within one of the more challenging modalities for such experiments. Nevertheless, allowing pigeons to "flutter" or training them to fly back and forth through different magnetic fields was not sufficient to permit discrimination of magnetic stimuli, as had been previously suggested (Bookman, 1977; Kreithen \& Keeton, 1974).

The two magnetic fields we used as stimuli were chosen for their similarity to those used by Bookman (1977), in that they differed mostly in their vertical intensity (see Table 1). Despite considerable care taken in the positioning of the flight tunnel within the laboratory space so as to obtain the most uniform ambient magnetic field within the tunnel, there was a residual spatial variation or gradient of magnetic field intensity that was similar under the two stimulus conditions. The average difference between the two magnetic field stimuli was 10 times larger than the variation within each stimulus due to this gradient. However, it is possible that this gradient may have prevented discrimination of the two stimuli, either because the spatial pattern of magnetic field intensity common to the two stimuli may have been more salient to the animal than the difference in mean vertical intensity between the stimuli or because the resultant variation interfered with a magnetic sense. Support for this interpretation comes from reports of disorientation of pigeons released at magnetic anomalies exhibiting comparable variation in magnetic intensity over much larger distances (Frei \& Wagner, 1976; Wagner, 1983; Walcott, 1978). Thus, the use of magnetic field stimuli that have either different spatial gradients and equal mean intensities or no gradients and different mean intensities might yield discrimination in future experiments.

The isolation and control of stimulus variables that can be achieved in the laboratory would be difficult, if not impossible, to duplicate in the field. However, such laboratory experiments may not adequately simulate the behavioral context of homing to be useful in exploring all the sensory mechanisms used in navigation. By holding other stimuli constant and restricting the range of behavior during magnetic field discrimination training, we present the animal with an impoverished environment that may not adequately approximate that encountered by the homing pigeon released at an unfamiliar site to motivate or permit the use of a navigational magnetic sense. Such use of magnetic cues in the field and their nonuse in the laboratory could be more a matter of unconscious reflex than of conscious choice on the part of the animal. If this were the case, it might be very difficult, if not impossible, for the animal to form associations between magnetic stimuli and arbitrary behaviors under laboratory conditions, a circumstance that also has its precedents (LoLordo, 1979). This interpretation suggests that it would not be possible to demonstrate discrimination under laboratory conditions, not because the animal could 
not sense magnetic fields, but rather because its use of magnetic information was restricted to navigational contexts.

If a magnetic sense exists in these animals, then our experiments either serve to constrain the conditions under which magnetic sensitivity can be demonstrated or suggest that such laboratory experiments may fail for reasons beyond experimental control. Although the failure to demonstrate discrimination of magnetic fields does not prove that a magnetic sense does not exist, our findings are also consistent with the absence of magnetic sensitivity in homing pigeons. There now are numerous reports of failure to demonstrate magnetic discrimination, whereas the only two claims of magnetic discrimination (Bookman, 1977; Reille, 1968) have not withstood replication. Regardless of whether or not these animals possess a magnetic sense, these results make it less likely that magnetic discrimination by these animals will be demonstrated under laboratory conditions. Therefore, it may be more appropriate to attempt to replicate and extend the field studies that suggested the existence of a magnetic sensitivity in homing pigeons in the first place.

\section{REFERENCES}

ALSOP, B. (1987). A failure to obtain magnetic discrimination in the pigeon. Animal Leaming \& Behavior, 15, 110-114.

Beaugrand, J. P. (1976). An attempt to confirm magnetic sensitivity in the pigeon, Columba livia. Journal of Comparative Physiology, 110, 343-355.

Brtterman, M. E. (1979). Discrimination. In M. E. Bitterman, V. M. LoLordo, J. B. Overmier, \& M. E. Rashotte (Eds.), Animal learning: Survey and analysis (pp. 413-443). New York: Plenum Press.

Bookman, M. A. (1977). Sensitivity of the homing pigeon to an earthstrength magnetic field. Nature, 267, 340-342.

Carman, G. J., \& MAHOWALD, M. (1984). [Attempted replication of M. A. Bookman's (1977) experiments.] Unpublished data.

Delrus, J. D., \& Emmerton, J. (1978). Sensory mechanisms related to homing in pigeons. In K. Schmidt-Koenig \& W. T. Keeton (Eds.), Animal migration, navigation, and homing (pp. 35-41). New York: Springer-Verlag.

FreI, U., \& WAgner, G. (1976). Die Anfangsorientienung von Brieftauben im erdmagnetisch gestörten Gebiet des Mont Jorat. Revue Suisse de Zoologie, 83, 891-897.

GellermanN, L. W. (1933). Chance orders of alternating stimuli in visual discrimination experiments. Journal of Genetic Psychology, 42, 206-208.
GRIFFIN, D. R. (1982). Ecology of migration: Is magnetic orientation a reality? Quarterly Review of Biology, 57, 293-295.

KeEton, W. T. (1971). Magnets interfere with pigeon homing. Proceedings of the National Academy of Sciences, 68, 102-106.

KREITHEN, M. L. (1978). Sensory mechanisms for animal orientationcan any new ones be discovered? In K. Schmidt-Koenig \& W. T. Keeton (Eds.), Animal migration, navigation, and homing (pp. 25-34). New York: Springer-Verlag.

Kreithen, M. L., Keeton, W. T. (1974). Attempts to condition homing pigeons to magnetic stimuli. Journal of Comparative Physiology, 91, 355-362.

LoLoRDo, V. M. (1979). Constraints on learning. In M. E. Bitterman, V. M. LoLordo, J. B. Overmier, \& M. E. Rashotte (Eds.), Animal learning: Survey and analysis (pp. 473-504). New York: Plenum Press.

Mackintosh, N. J. (1974). The psychology of animal learning. New York: Academic Press.

McIsaAC, H. P., Kreithen, M. L. (1987). Attempts to condition homing pigeons to magnetic cues in an outdoor flight cage. Animal Learning \& Behavior, 15, 118-123.

MeYer, M. E., \& LAMBE, D. R. (1966). Sensitivity of the pigeon to changes in the magnetic field. Psychonomic Science, 5, 349-350.

Moore, B. R., Stanhope, K. J., \& Wilcox, D. (1987). Pigeons fail to detect low-frequency magnetic fields. Animal Learning \& Behavior, $15,115-117$.

ORGEL, A. R., \& SMITH, J. C. (1954). Test of the magnetic theory of homing. Science, 120, 891-892.

OSSENKOPP, K.-P., \& BARBEITo, R. (1978). Bird orientation and the geomagnetic field: A review. Neuroscience \& Biobehavioral Reviews, $2,255-270$.

Reille, A. (1968). Essai de mise en évidence d'une sensibilité du pigeon au champ magnétique à l'aide d'un conditionnement nociceptif. Joumal de Physiologie (Paris), 60, 85-92.

WAGNER, G. (1983). Natural geomagnetic anomalies and homing in pigeons. Comparative Biochemistry \& Physiology, 76A, 691-700.

WALCOTT, C. (1978). Anomalies in the earth's magnetic field increase the scatter of pigeons' vanishing bearings. In K. Schmidt-Koenig \& W. T. Keeton (Eds.), Animal migration, navigation, and homing (pp. 143-151). New York: Springer-Verlag.

W ALCotT, C., \& GReEN, R. P. (1974). Orientation of homing pigeons altered by a change in the direction of an applied magnetic field Science, 184, 180-182

WoODARD, W. T., \& BiTterman, M. E. (1974). A discrete-trials/fixedinterval method of discrimination training. Behavior Research Methods \& Instrumentation, 6, 389-392.

(Manuscript received December 11, 1985; revision accepted for publication July 1,1986 .) 